\title{
Study on Impact of Big Data in User Experience Practices
}

\author{
Chun-Hui Chen \\ Education Technology Center \\ Guangdong University of Foreign Studies \\ Guangzhou, China \\ 247031690@qq.com
}

\author{
Ke-Jing Li \\ School of Business \\ Guangdong University of Foreign Studies \\ Guangzhou, China \\ ljh@oamail.gdufs.edu.cn
}

\begin{abstract}
Users are heart of all enterprise activities and data is the core of all enterprises' decision-making. The development trend of big data has subverted the research mode, extended the scope, and improved the overall quality of user experience research. Based on the needs of the Internet industry to study user experience, this paper combines the relevant experience in the development of computer science and psychology, and summarizes the big data in the field of user experience into four categories (structured data, text data, multimedia and network behavior data) to introduce data analysis methods. It also indicates two practical circumstances where user experience practices are enabled by big data technology and provides directional guidance on its application. Finally, the paper focuses on the user privacy implications of user experience research under network big data.
\end{abstract}

Keywords—Network Big Data; User experience; Application; Practices; Psychology

\section{INTRODUCTION}

With the rapid development of the Internet, a large number of social media users have posted their daily life records on the Internet. These records are a natural reflection of the users' psychological characteristics and activities. The characteristics of rich content, natural occurrence, large scale and convenient backtracking make the social media platform an ideal "online psychology laboratory" [1]. The product big data obtained by the online platform has the characteristics of good ecological effects, low cost, and large scale, which can directly improve the efficiency of user experience research and enhance the validity of the research. However, from the current research on big data and user experience, since they both are novel concepts, the independent research results are still limited, and the joint research on the two concepts can hardly be found.

This study describes the technical characteristics of network big data development from the perspective of application and points out the important influence and promotion of big data on the user experience research logic and methods. Based on this, it tries to transplant the big data plan in the research of social psychology to the emerging research field of user experience and summarizes the user experience research methodology from the perspective of data analysis, aiming at promoting the practicality and operability of the increasingly mature technology of network big data in different industries and at all levels of user experience, and at providing a new perspective for user experience research.

\section{Classification AND APPliCATION OF Network Big Data FROM THE PERSPECTIVE OF USER EXPERIENCE}

It is generally believed that the user experience includes three factors: user, product or service, and interaction environment. The product element is relatively stable, but users and the interaction process between the users and the product has a strong subjective feeling brought by individual differences. If the user is viewed as an individual, the measurement method of individual emotions, perceptions, cognitions and so on in psychology can be used to disassemble and analyze the user experience. The measurement and evaluation of user experience can be influenced by individual characteristics such as different demand preferences and cultural backgrounds. Therefore, research on user experience measurement should take more into account the influence of user background. In addition, researchers generally believe that the user experience has the characteristics of environmental dependence [2]. The user background not only includes the user's past experience but should also be placed in the specific current interactive environment: differences between real and virtual environments, mobile and fixed device, interactions between users in an interactive environment, and other interactive tasks that occur at the same time affecting the interactive experience.

With the popularization of the Internet, the research on user experience under the network environment has received great attention. However, due to the fact that the actual demand is large, research is usually carried out by individual enterprises in response to their own business needs. Social psychology places emphasis on the influence of the environment on people's attitudes and behaviors, which shares some common concepts with user experience. In contrast, the academic community has more in-depth research on big data-directed social psychology. Therefore, selective reference and transplantation of research methods and results can accelerate the research process of big data affecting user experience, achieving knowledge sharing and development for all in crosscutting areas. 
In the field of big data application, the data categories involved in psychosocial and behavioral analysis of social groups can be divided into structured and unstructured data. According to different manifestations, Unstructured data can be further subdivided into text data, multimedia data and network behavior data, as shown in Fig.1.

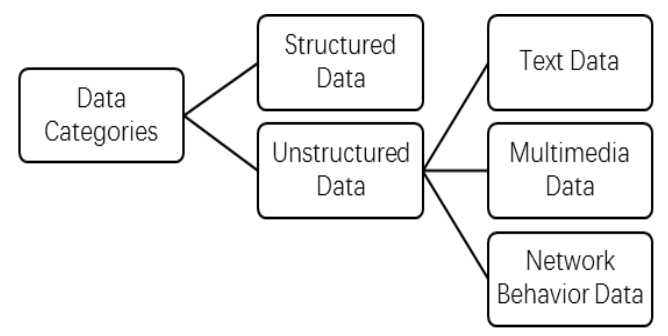

Fig. 1 Data Categories

\section{A. Structured Data}

Traditional data analysis mainly focuses on structured data. Structured data has predefined data models (determined length and structure) and existing computer database technologies can meet most of the organization, searching, and analysis needs. Thanks to the development of relational database technology, the analysis method of structured data is relatively mature, and most of them are based on data mining and statistical analysis [3].

\section{B. Unstructured Data}

Compared to structure data that records production, business, transaction, and customer information, unstructured information covers a wider range of content, including operational content such as contracts, invoices, letters, and procurement records; departmental content such as word processing e-form spreadsheets, newsletter archives, and emails; Web content such as HTML and XML; and media content such as sound, video and graphics. According to the IDC survey report in 2011, unstructured data will account for $90 \%$ of the new data generated in the next decade. With the continuous development of Internet technology, the scale and growth rate of unstructured data are exceeding structured data with a speed beyond imagination, and database technology has accordingly entered the "post-relational database era." A huge information base about corporate opportunities and fate contained in unstructured data and unmatched analysts and technologies give the field unlimited value and potential to enhance the user experience.

1) Text Data: Text data is the most common type of unstructured data and generally contains network information stored and transmitted in text form. Text Mining, also often referred to as Text Data Mining or Knowledge Discovery in Texts, uses unstructured language texts as mining samples, often referring to the process of applying mining technology to discover implicit, unknown, potentially valuable information from large-scale text collections [4].
2) Multimedia Data: Multimedia data mainly includes images, audio, and video on various network platforms. Compared with text data, the types of multimedia data are complex and diverse, with large differences between types and rich semantics. They are the most difficult type to analyze among the unstructured data. Multimedia analysis covers a wide range of research, including multimedia abstraction, multimedia annotation, multimedia indexing and retrieval, multimedia recommendation, and multimedia event detection. In the future, user experience researchers can consider working with computer talents to achieve intelligent identification and analysis.

3) Network Behavior Data: The fact that cannot be ignored in the information age is that all actions of users on the Internet are being tracked and recorded every moment. In addition to tangible data sources such as text and multimedia data, the network behavior data also includes traces of intangible behavior data left over by users of the network. The Internet using behavior specifically refers to a large number of clickstreams, transaction data, and user model data generated during the use of the Internet by a user, including server log files (user request time, client IP address, requested resource, and so on.) and user statistics (browsing history, purchase history, ratings, and so on.)[5]. This type of data has a wide range of sources and diverse types The format is simple and directly reflects the user's interactive behavior. It is easier to capture data meaning than text data and multimedia data. Based on the data analysis of this type, product developers can more intuitively find the current weak links in design to improve the effectiveness and timeliness of adjustments. Usually different companies have different behavioral indicators that are of concern to each other. For example, ecommerce platforms pay attention to users' purchase behaviors, video websites pay attention to users' browsing behaviors, search engines pay attention to users' search behaviors. However, some universally applicable indicators that illustrate certain problems are often overlooked, such as the user's IP, user clicks and stops on the page.

\section{BIG DATA ENABLED PRACTICES}

\section{A. Product development and design}

1) Innovative product development: User experience designers can use the user's big data to remember and identify the user's usage habits and behavioral characteristics, guide the innovation of products and services based on historical data mining and big data support and develop focused, distinctive products and services to meet the targeted customers' needs. The construction of user experience model starting from the product's usability and durability and the detection and comparison of key user experience indicators through the collection and analysis enabled by big data technology, can create new products with high user fit. 
2) User-centered design: Product design thinking centered on users and their data is the essence of current Internet product development. This idea runs through the whole process of product development and design process, and is characterized by interactivity, emotionality and feedback. The thinking mode is mainly manifested in the following aspects in practice:

a) Interactivity: The interactive design aims at the experiential style, continuously improve the user's active participation behavior in the interaction between the user and the product through interactive technology. The users are stimulated sensually and psychologically in a variety of interactive processes such as participation in operations., obtaining a higher level of user experience.

b) Emotionality: Fully consider the emotional needs of the group and the individual in the design process and realize the user's emotion expectation to the product and the psychological state fluctuation in the change of the product function, especially the different visual performances.

c)Feedback: Design is not a once-in-a-lifetime matter, but an innovation in the continuous cycle. A complete feedback mechanism should be established during and after the design process to form a closed-loop cyclic design.

3) Differentiated intervention: Analyze the relationship between customers' conversion and churn rate and other users' behavior or attitude data in each layer of the tasks during the operation of an existing product or a new product. Improve users' experience and perception by analyzing the parts that need to be optimized in each design detail. Timely and appropriate differentiated interventions are important strategies for maintaining user relationships, helping to identify moving users and greatly enhancing the user loyalty after improvements.

\section{B. Two-way network resource communication}

Faced with huge network information resources, the essence of user experience lies in the two-way information exchange between users and systems. The quality of information communication determines the quality of user experience. Under the influence of modern interactive design thinking, the user's role has changed dramatically from passive acceptance of data to active acquisition, exploration, creation and control of analytical data. This change has made user behavior detection a key step in user experience design and requires the user experience system designer to fully consider every interaction in relation to decision making of the users.

1) User passive reception: The information that the users passively accept mainly refers to the part of the information that is selectively transmitted and presented by the enterprise. The efforts that companies can make in information communication can be divided into two aspects: content and format.

a) In terms of content: Achieving precise positioning and marketing through multi-angle analysis and modeling identification of user information, company can promote different products to different users to enhance the current user experience and increase the probability of success for the next interaction. Designers are able to deeply mine the special needs of users and create a personalized user interface to maximize the value of different users based on data.

b) In terms of format: To enhance the efficiency and effectiveness of data transfer, data visualization can be used to attract users' attention by turning complex data into more comprehensible visual elements such as images, videos, audio, and so on., reducing the difficulty and threshold for users to accept. Visualization should be diversified in terms of presentation, such as using the latest 3D technology to make data more stereoscopic and specific. It is worth noting that when users can actively manipulate the visualization set according to their own needs, the information communication result achieved is often better.

2) User active request: When the users actively request the server data service, the important evaluation indicators that affect the quality of the users' perceived service include response time, accuracy, interestingness, and so on.

a) Shorten response time: Through powerful computing and storage capabilities, the router can discover the best content location of the user experience based on the content requested by the user, the location, environment and devices used, and obtain and cache content to improve the utilization of network resources. [6]

b) Improve accuracy: Real-time mobilization and reading of massive data will happen by the cross-platform data integration and analysis and the rapid processing capability of massive data of big data technology, satisfying users' diversified requests [7].

c)Strengthen interestingness: When the user's request is responded, the presentation of the response can be of interest to the users and encourage the users' interaction behaviors. For example, the recent occurrence of user participation in generation of big data summary reports has promoted social media awareness while enhancing user experience perception.

\section{Future RESEARCH PROSPECTS}

When big data is widely used in business analysis and decision-making, there is a paradox worthy of discussion between user experience and user privacy. If large commercial organizations or departments take a monopolistic approach to data, it will inevitably cause some degree of damage to the interpretation of data sources and affect the efficiency of data mining. Therefore, in order to improve the validity of the analysis conclusions and further optimize the user experience, obtain as complete and comprehensive data as possible is the premise and key to big data analysis. On the other hand, improper use of personal data, especially through the connection of data from multiple sources, can harm personal privacy and endanger the customer's personal rights. With more and more transparent personal information, it has also been widely discussed whether data collectors have the right to collect, analyze, and use relevant information. To avoid the moral and ethical issues that accompany the development of science and technology, business researchers should conduct 
valuable research under conditions that fully guarantee the privacy of users. For some data that are not relevant to research, encryption or anonymization can be used. At the same time, the state should also embark on the construction and improvement of laws related to information security.

\section{SUMMARY}

Traditionally, user experience research is mainly the work of psychologists and designers. However, in recent years, engineering talents such as computer and automation have emerged. This is a confirmation of the current need for technical support for user experience research. People are the center of user experience research and human's needs and wishes are also the source of all scientific and technological progress. Human-oriented, cross-disciplinary integration, data resource utilization, and data visualization are the future of big data development. Big data itself is the product of technological development. To achieve the development of science and technology, we must also seek the support of science and technology. A large number of data samples face the problems of collection, storage, processing, and analysis that require modern computer technology. As the focus of the development of big data technology, data mining's core stepdata modeling is the hot spot in the current research in computer field, using statistical analysis and machine learning methods to analyze and model the data. "Specialists" can no longer meet the development needs of user experience. Crossdisciplinary talents can open up blind spots and continue to provide new innovations and breakthroughs for research.

\section{REFERENCES}

[1] N. Zhao, B. B. Hao, "Psychological Character Modeling Based on Online Big Data: thePrediction of Personality, Mental Health, and Subjective Well-Being", Summary of the 18th national psychological academic conference, pp.619, 2015. (In Chinese)

[2] Y. Ding, F. Guo, M. C. Hu, F. L. Sun, "A Review of User Experience", Industrial Engineering and Management, Vol. 04, pp. 92-97+114, 2014. (In Chinese)

[3] Y. Zhang, M. Chen, X. F. Liao, "Big Data Applications: A Survey", Journal of Computer Research and Development, China, Vol. 2, pp. 216-233, 2013. (In Chinese)

[4] I. V. Mashechkin, M. I. Petrovskiy, D. S. Popov, D. V. Tsarev, "Applying text mining methods for data loss prevention", Programming and Computer Software, Russian, Vol. 41, No. 1, pp. 23-30, January 2015. In English.

[5] Y. S. Li, Z. W. Huang, X. Y. Tan, L. Liu, "Cultural difference and cultural change in internet data", Advances in Psychological Science, Vol. 06, pp. 1045-1057, 2017. (In Chinese)

[6] H. Yin, B. Qiao, "Big data-driven network information plane", Chinese journal of computers, 39 vol, page 126-139, 2016. (In Chinese)

[7] T. S. Zhu, J. Y. Wang, N. Zhao, X. Q. Liu, "Reform on Psychological Research in Big Data Age", Journal of Xinjiang Normal University (Philosophy and Social Sciences), China, vol 04, pp. 100-107+2, 2015. (In Chinese) 